Article

\title{
Design and Fabrication of Full Wheatstone- Bridge-Based Angular GMR Sensors
}

\author{
Shaohua Yan ${ }^{1}$, Zhiqiang Cao ${ }^{2}$, Zongxia Guo ${ }^{2}$, Zhenyi Zheng ${ }^{1}$, Anni Cao ${ }^{1}$, Yue Qi ${ }^{3}$, \\ Qunwen Leng ${ }^{2,4, *}$ and Weisheng Zhao ${ }^{1,2, *}$ \\ 1 Fert Beijing Institute, BDBC, School of Electronic and Information Engineering, Beihang University, \\ Beijing 100191, China; yanshaohua@buaa.edu.cn (S.Y.); zhenyizheng@buaa.edu.cn (Z.Z.); \\ caoan@buaa.edu.cn (A.C.) \\ 2 Beihang-Goertek Joint Microelectronics Institute, Qingdao Research Institute, Beihang University, \\ Qingdao 266000, China; zhiqiangcao@buaa.edu.cn (Z.C.); gzx0106@buaa.edu.cn (Z.G.) \\ 3 State Key Laboratory of Virtual Reality Technology and Systems, Beihang University, \\ Beijing 100191, China; qy@buaa.edu.cn \\ 4 Goertek Inc., Weifang 261031, China \\ * Correspondence: lengqw@bhqditi.com (Q.L.); weisheng.zhao@buaa.edu.cn (W.Z.); \\ Tel.: +86-532-5866-6313 (Q.L.); +86-10-8231-4875 (W.Z.)
}

Received: 28 April 2018; Accepted: 1 June 2018; Published: 5 June 2018

\begin{abstract}
Since the discovery of the giant magnetoresistive (GMR) effect, GMR sensors have gained much attention in last decades due to their high sensitivity, small size, and low cost. The full Wheatstone-bridge-based GMR sensor is most useful in terms of the application point of view. However, its manufacturing process is usually complex. In this paper, we present an efficient and concise approach to fabricate a full Wheatstone-bridge-based angular GMR sensor by depositing one GMR film stack, utilizing simple patterned processes, and a concise post-annealing procedure based on a special layout. The angular GMR sensor is of good linear performance and achieves a sensitivity of $0.112 \mathrm{mV} / \mathrm{V} / \mathrm{Oe}$ at the annealing temperature of $260{ }^{\circ} \mathrm{C}$ in the magnetic field range from -50 to +50 Oe. This work provides a design and method for GMR-sensor manufacturing that is easy for implementation and suitable for mass production.
\end{abstract}

Keywords: GMR effect; angular sensor; full Wheatstone bridge; synthetic antiferromagnet

\section{Introduction}

Giant magnetoresistive (GMR)-based magnetic sensors have attracted much attention since the GMR effect was discovered by Albert Fert and Peter Grünberg in the 1980s [1,2]. Compared to their counterparts anisotropic magnetoresistance (AMR) or Hall sensors, GMR sensors display greater sensitivity and lager linear range [3] and have wide applications in compasses, electrical current measurement, detection of biologic antibodies labeled by magnetic beads, or nondestructive testing [4-8]. A special type of GMR structure is the spin valve, which was proposed by Dieny [9] and consists of two ferromagnetic layers separated by a nonmagnetic conductor spacer, usually made of $\mathrm{Cu}$. One of these layers is pinned by an antiferromagnetic layer such as IrMn or PtMn, while the other is free to rotate. The pinned layer could be replaced by a synthetic antiferromagnetic (SAF) structure to diminish the mutual magnetostatic coupling between the free and pinned layer [10,11].

For sensor application, a linear output is required, the easy axis of the pinned layer is usually arranged in the transverse orientation of a patterned stripe, and the easy axis of the free layer is in the longitudinal orientation [12]. In order to avoid output fluctuation caused by temperature drift, a Wheatstone bridge configuration is needed. However, if each of the MR elements has the same dimension and magnetization direction, each arm in the bridge responds identically to an applied field 
and the bridge generates no differential output. To make the bridge operational, a full Wheatstone bridge (see Figure 1) is very useful, in which two of bridge arms exhibit $\mathrm{dR} / \mathrm{dH}>0$, while the remaining two exhibit $\mathrm{dR} / \mathrm{dH}<0$ [13].

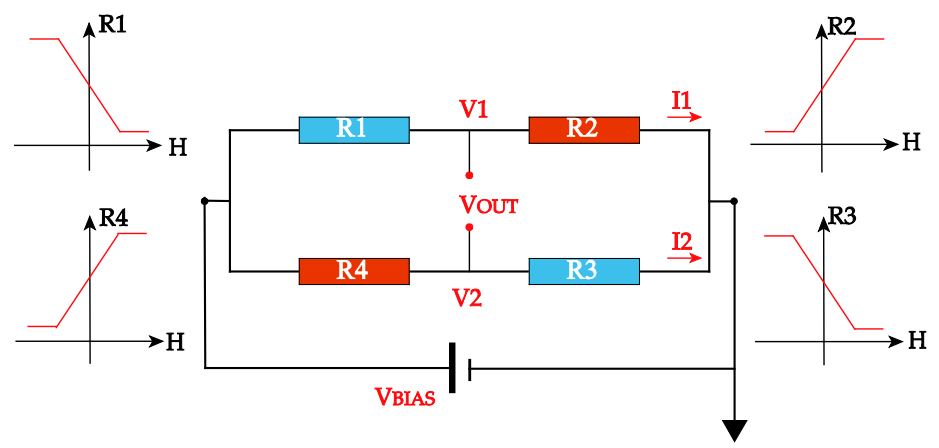

Figure 1. Schematic of a full bridge GMR sensor. It exhibits a null output in the absence of a signal field and offers an intrinsic compensation for thermal drift. When an external field $\mathrm{H}$ is applied, the bridge has a linear output. $R_{1,3}(H)=R-\triangle R(H) ; R_{2,4}(H)=R+\triangle R(H) ; V_{\text {out }}=V_{\text {bias }}{ }^{*} \Delta R / R$.

Several methods have been reported for the realization of a full bridge sensor. The simplest solution is to mechanically assemble GMR elements along the same direction but in opposite senses, which has important drawbacks such as alignment errors and is not suitable for mass production [13]. Another way is to deposit two stacks on different regions of the same wafer, and the magnetic fields used for two depositions are antiparallel. This is also a sophisticated process. Alternatively, after the fabrication of the bridge sensor, a local magnetic field annealing over each bridge element is done by a current flowing through the overlaying setting circuit to realign the magnetization of pinned layer $[12,14]$. The current flows in opposite directions for two pairs of resistors in the bridge. The amplitude of the current should be carefully calculated and a particular PCB is needed. A similar way is to use laser radiation in an applied field in place of the setting circuit $[15,16]$. However, all these methods are more or less complex.

In the present work, we demonstrated a concise experimental method for the realization of a full bridge GMR sensor using one GMR film stack. On the basis of a special layout, only a one-time post annealing procedure is required. In the following paragraphs, the details of this method as well as the characterization results are described.

\section{Materials and Processes}

A thin film stack with a structure of $2.0 \mathrm{Ta} / 3.0 \mathrm{Ru} / 7.0 \mathrm{IrMn}_{80} / 2.0 \mathrm{CoFe}_{10} / 0.85 \mathrm{Ru} / 2.1 \mathrm{CoFe}_{10} / 1.9$ $\mathrm{Cu} / 1.2 \mathrm{CoFe}_{10} / 2.5 \mathrm{NiFe}_{19} / 4.0 \mathrm{Ta}$ (numbers denote layer thickness in $\mathrm{nm}$ ) was deposited on $\mathrm{SiO}_{2}$ substrate with a base pressure less than $10^{-8}$ Torr by a Singulus ROTARIS ultra-high vacuum magnetron sputtering system. The $\mathrm{CoFe}_{10} / \mathrm{Ru} / \mathrm{CoFe}_{10}$ part constituted a synthetic antiferromagnetic structure (see Figure 2a). The layout of the bridge sensor is illustrated in Figure 2b. The microelectronic fabrication process of the devices consisted of three lithographic steps. In the first one, the contact leads and pads were defined, using $10 \mathrm{~nm} \mathrm{Cr} / 80 \mathrm{~nm}$ Au by E-beam evaporation. Then, the GMR stripes were patterned by photolithography followed by ion-beam etching into active sensing elements with dimensions of $3 \times 225 \mu \mathrm{m}$. The final passivation layer consisted of $80 \mathrm{~nm} \mathrm{SiO}_{2}$ film by E-beam evaporation. The device was annealed in a vacuum at $250{ }^{\circ} \mathrm{C}$ under a negative field of $0.9 \mathrm{~T}$ along the $x$-axis for $1 \mathrm{~h}$. Then, the field was changed to positive $50 \mathrm{mT}$ for $15 \mathrm{~min}$ before cooling down without the field applied. Note that there was an angle of 45 degrees between the annealing direction and the longitudinal orientation of the GMR stripes. The R-H loops of the bridge arms and voltage output of the bridge were measured by a standard four-probe method at room temperature. 


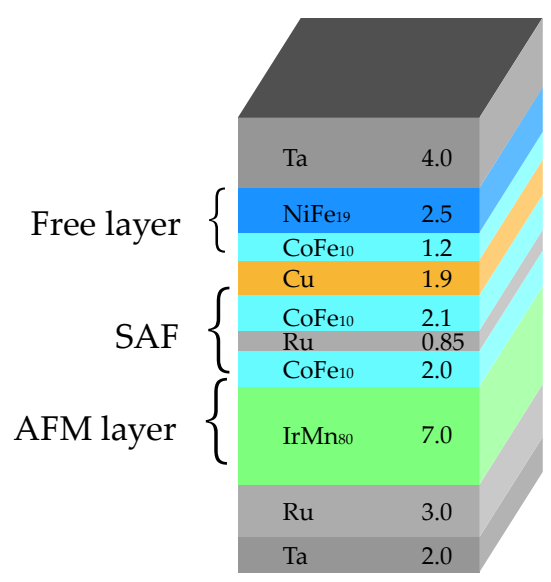

(a)

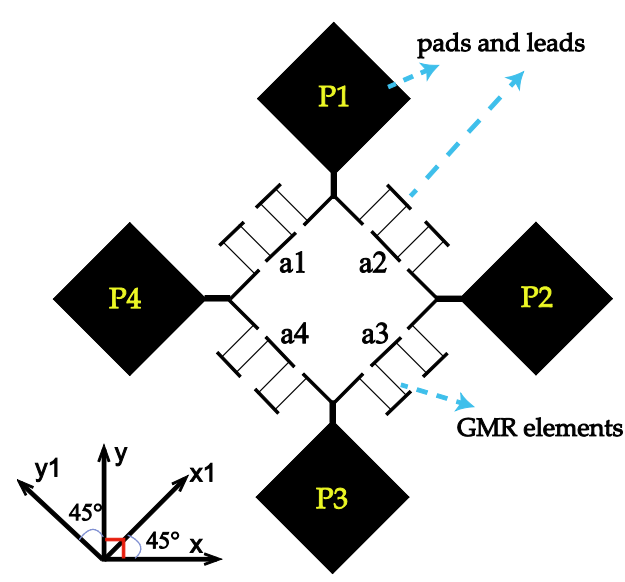

(b)

Figure 2. (a) Structure of GMR stack with SAF CoFe $\mathrm{C}_{10} / \mathrm{Ru} / \mathrm{CoFe}_{10}$. (b) Layout of Wheatstone bridge sensor. P1-P4 are four electrodes of the bridge used for electrical measurements. Each bridge arm (a1-a4) consists of four GMR stripes with dimensions of $3 \times 225 \mu \mathrm{m}$ connected in series. The meander connection of four stripes is used to obtain the resistance as designed in the bridge.

\section{Results and Discussion}

The R-H loops of each bridge arm are measured separately. A $1 \mu \mathrm{A}$ DC current was used while applying an external field in different directions. The field utilized was swept from -300 Oe to +300 Oe and back to -300 Oe by a step of 6 Oe. Figure 3a illustrates that after annealing, the four arms of the bridge behave almost the same to the external field along the $x$-axis, where the output of the bridge should be null. The observed GMR ratio is $5.25 \%$. It should be noted that the maximal intrinsic GMR ratio will be larger because the magnetization directions between the free and pinned layers are not antiparallel and parallel within the applied field. As the R-H loops have an offset from zero and enter the state of high resistance before $\mathrm{H}_{\mathrm{x}}=0 \mathrm{Oe}$, the magnetization of the free layer $\mathrm{CoFe}_{10} / \mathrm{NiFe}_{19}$ is antiferromagnetically coupled with the pinned layer across the spacer layer $\mathrm{Cu}$. The coupling field $\mathrm{H}_{\text {in }}$ is about -20 Oe. For sensor application, a weak or no interlayer coupling is desired and it could be adjusted by tuning the layer thicknesses [17].

Figure $3 \mathrm{~b}$ shows that arm 1 and arm 3 exhibit $\mathrm{dR} / \mathrm{dH}>0$, while arm 2 and arm 4 exhibit $\mathrm{dR} / \mathrm{dH}<0$. This conforms to the characteristics of a full bridge sensor along the $y$-axis. The resistance of the GMR element depends on the angle $\alpha$ between the free and pinned layers magnetization [18], described by

$$
R(\alpha)=R_{p}+\left(R_{a p}-R_{p}\right)(1-\cos \alpha) / 2
$$

where $R_{p}$ and $R_{a p}$ are the resistances of parallel and antiparallel alignments of the free and pinned layers magnetization in the GMR element. The variation of resistance results from the rotation of free layer magnetization.

The R-H loops in Figure $3 \mathrm{~b}$ are not symmetric around the $y$-axis, so the pinning direction is not perpendicular to the $y$-axis. We note the angle between pinned layer easy axis and $y$-axis as $\theta$. To estimate $\theta$, we suppose that our device stays in the single-domain state and the magnetic reversal occurs by coherent rotation of magnetization. At point A in Figure $3 c$, the external field $\mathrm{H}=0$ Oe. Due to the antiferromagnetic coupling between the free and pinned layer, their magnetizations are antiparallel, and the element has the highest resistance. At points $B$ and $C$, the free layer magnetization follows the direction of the applied field. Using Equation (1), we obtain

$$
\frac{R_{A}-R_{B}}{R_{A}-R_{C}}=\frac{1+\cos (\pi-\theta)}{1+\cos \theta}=\frac{\cos ^{2}\left(\frac{\pi-\theta}{2}\right)}{\cos ^{2}\left(\frac{\theta}{2}\right)}=\tan ^{2}\left(\frac{\theta}{2}\right)
$$


Then the angle $\theta$ can be expressed as

$$
\theta=2 \arctan \sqrt{\frac{R_{A}-R_{B}}{R_{A}-R_{C}}}
$$

The calculated result of $\theta$ for arm 1 in the bridge is $71.6^{\circ}$. Same calculations are performed for the other arms, and these angles are $106.7^{\circ}, 72.1^{\circ}$, and $107.1^{\circ}$, respectively, as shown in Figure $3 \mathrm{~d}$.

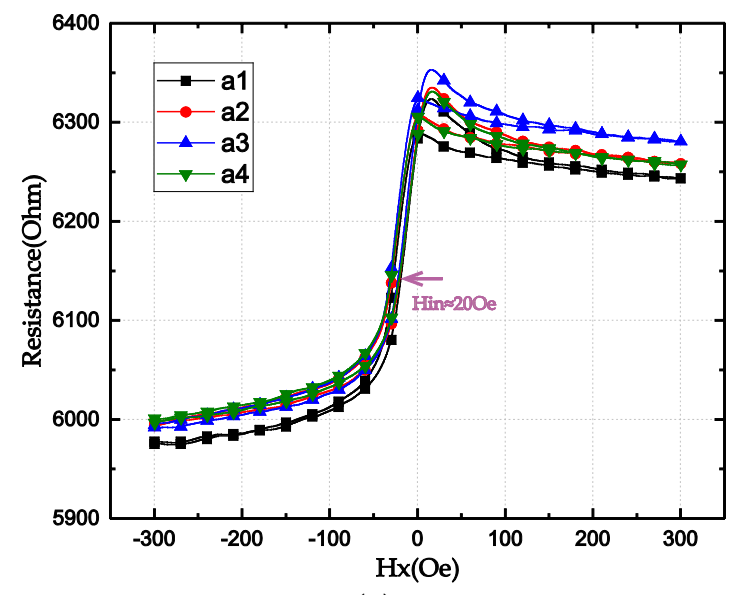

(a)

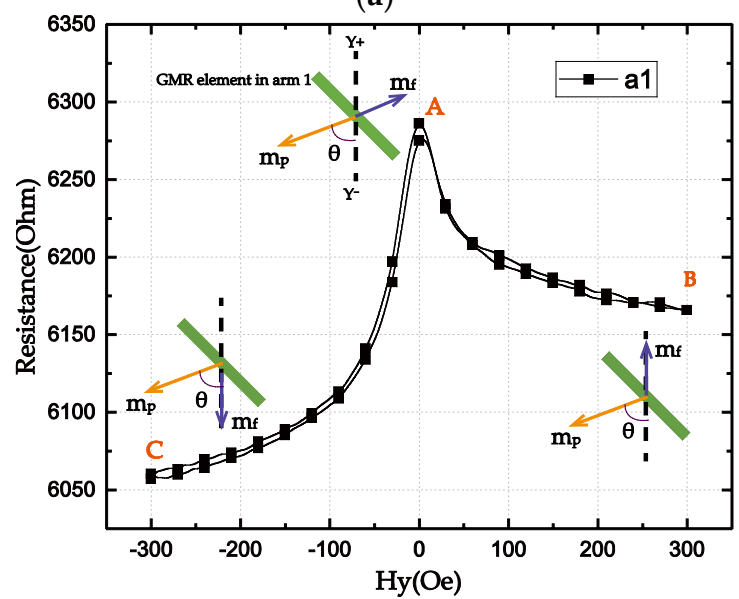

(c)

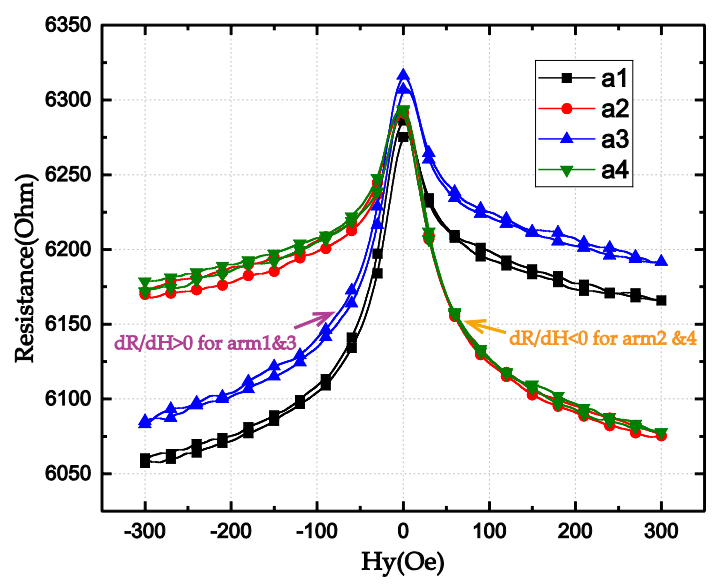

(b)

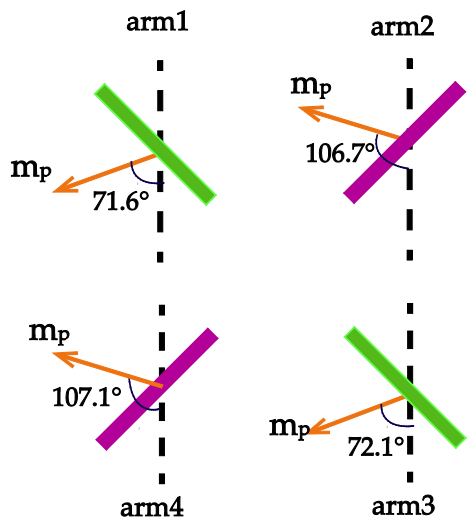

(d)

Figure 3. (a,b) R-H loops of the four bridge arms with the applied magnetic field along the $x$-and $y$-axes. (c) R-H loop of arm 1 with the applied field along the $y$-axis. $\mathrm{m}_{\mathrm{f}}$ and $\mathrm{m}_{\mathrm{p}}$ denote the magnetization of the free and pinned layers. (d) Calculated deflection angles of pinned layer magnetization in GMR elements.

Figure 4 shows the output of the full Wheatstone-bridge-based angular GMR sensor under bias voltage of $3 \mathrm{~V}$. The angular sensor is designed to be only sensitive to the external field in the $y$-axis, i.e., the $y$-axis is the sensing direction. The response in the $x$-axis is almost a constant. The voltage offset is due to the different resistances and pinning directions of four resistors in the bridge. The sensitivity of this sensor measures $0.09 \mathrm{mV} / \mathrm{V} / \mathrm{Oe}$ in the sensing axis. The bridge output as a function of the applied field angle is shown in Figure 5, where the magnitude of the field is fixed as 50e. A unique angle can only be determined between 0 and 180 degrees. 


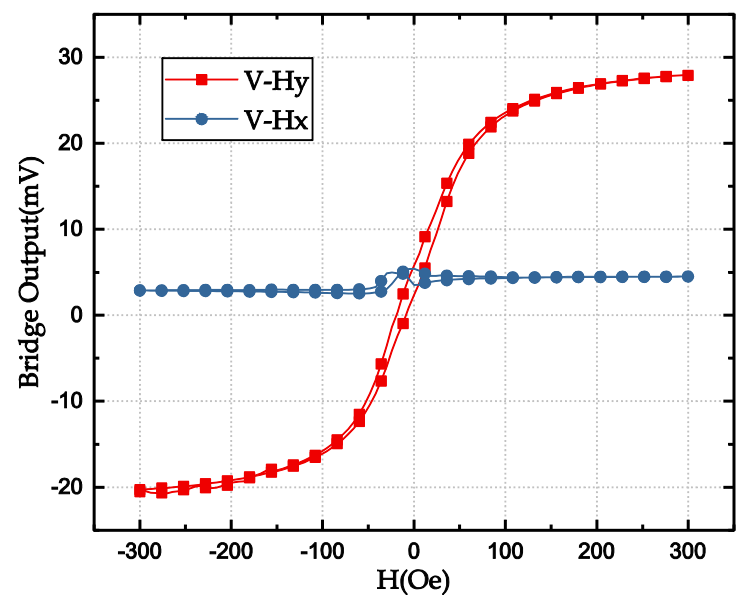

Figure 4. The output of the full Wheatstone-bridge-based GMR sensors under $3 \mathrm{~V}$ bias voltage in the $\mathrm{x}$ and $y$-axes.

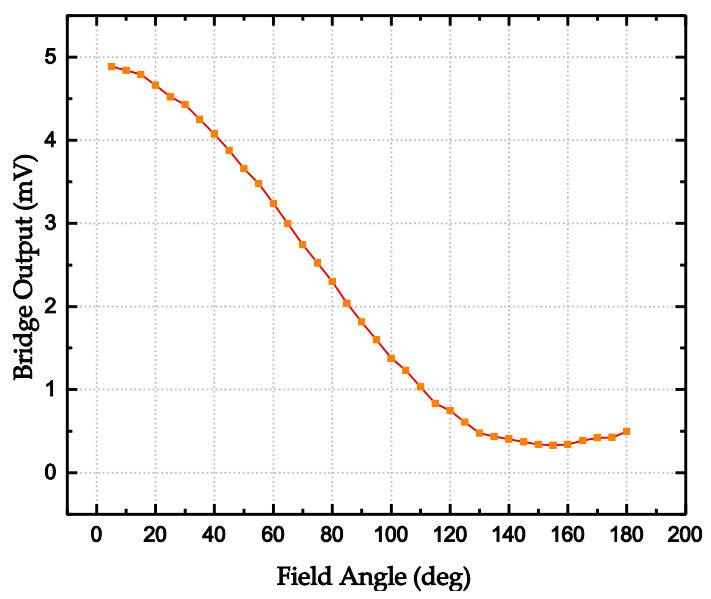

Figure 5. Bridge output as a function of the applied field direction.

The outputs of the full bridge-based sensors in the sensing axis at different annealing temperatures are demonstrated in the Figure 6 . The performances of the full bridge sensors annealed at different temperatures are listed in Table 1 for comparison. The magnetothermal process will change the sensor sensitivities that show an increase with the increases of the annealing temperature monotonically. The angle between the pinned magnetization and the $y$-axis should be $45^{\circ}$ and $135^{\circ}$ for bridge arms 1,3 and 2, 4, respectively. The more the magnetization of the pinned layer is away from these angles, the less the sensitivity of the bridge sensor is. The GMR ratio is improved at higher annealing temperature, and the sensitivity is as well. It can be seen that the linearity of the bridge response is better at the higher annealing temperature, as indicated in Figure 6.

Table 1. The performance of the full bridge-based GMR sensors at different annealing temperatures.

\begin{tabular}{cccc}
\hline $\begin{array}{c}\text { Annealing } \\
\text { Temprature }\end{array}$ & $\begin{array}{c}\text { Sensitivity } \\
(\mathbf{m V / V} / \text { Oe) }\end{array}$ & $\begin{array}{c}\text { Angles of Pinned Layer } \\
\text { Magnetization in Arm1 }\end{array}$ & $\begin{array}{c}\text { MR Ratio of } \\
\text { individual Element }\end{array}$ \\
\hline $260^{\circ} \mathrm{C}$ & 0.112 & $71.7^{\circ}$ & $6.12 \%$ \\
$250^{\circ} \mathrm{C}$ & 0.093 & $71.6^{\circ}$ & $5.25 \%$ \\
$240^{\circ} \mathrm{C}$ & 0.074 & $80.5^{\circ}$ & $5.36 \%$ \\
\hline
\end{tabular}




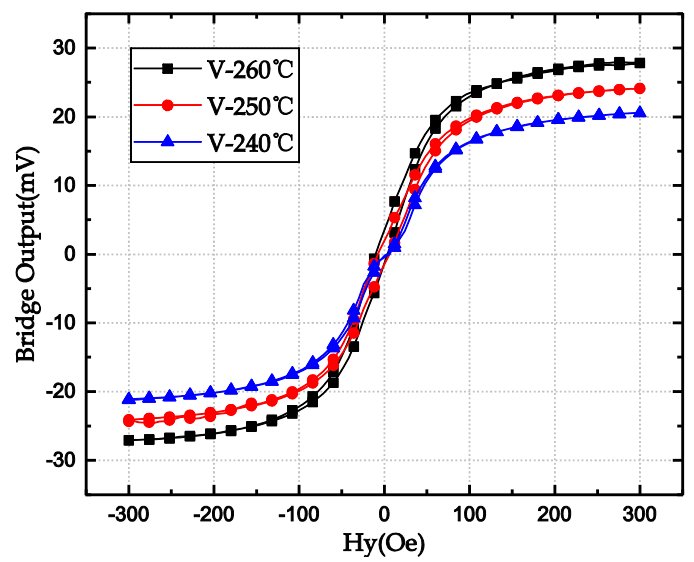

Figure 6. The outputs in the $y$-axis for the full Wheatstone-bridge-based GMR sensors annealed at different temperatures.

During the annealing process, the exchange coupling between $\mathrm{IrMn}$ and $\mathrm{CoFe}(\mathrm{P} 1)$ disappears when the temperature is higher than the blocking temperature of IrMn. With a field of $-0.9 \mathrm{~T}$ applied in the $x$-axis, the exchange coupling field direction of $\operatorname{IrMn} / \mathrm{CoFe}(\mathrm{P} 1)$ is set. After the field is removed, due to strong antiferromagnetic coupling in SAF and IrMn/CoFe, the magnetization of $\mathrm{CoFe}(\mathrm{P} 2)$ tends to align itself antiparallel to that of $\mathrm{CoFe}(\mathrm{P} 1)$, as indicated in Figure 7a. The annealing under the field of $50 \mathrm{mT}$ will barely impact the direction of the exchange coupling field because the magnetic field threshold for spin flop in SAF is about $100 \mathrm{mT}[18,19]$.



(a)

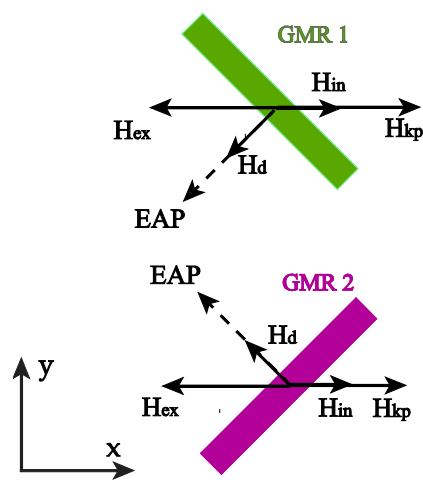

(b)

Figure 7. (a) Schematic of the exchange coupling field in SAF structure. (b) Direction of the pinned layer $\mathrm{CoFe}_{10}(\mathrm{P} 2)$ easy axis (EAP) after annealing.

As shown in Figure 7b, in the GMR element 1, the easy axis of its pinned layer $\mathrm{CoFe}(\mathrm{P} 2)(\mathrm{EAP})$ is defined by the competition among the demagnetizing field $\mathrm{H}_{\mathrm{d}}$, the exchange coupling field $\mathrm{H}_{\mathrm{ex}}$ from $\mathrm{SAF}$, the interlayer coupling field from free layer $\mathrm{H}_{\mathrm{in}}$, and the induced anisotropy field $\mathrm{H}_{\mathrm{kp}}$ [20]. Here, the short-time low-field annealing contributes to the induced anisotropy, taking effect to compensate the deviation of the easy axis direction. The ideal direction is along the transverse orientation of the patterned stripe.

The easy axis of the free layer is determined by the small interlayer coupling field $\mathrm{H}_{\mathrm{in}}$, the induced anisotropy field $\mathrm{H}_{\mathrm{kf}}$, and the demagnetizing field $\mathrm{H}_{\mathrm{d}}$, which make it tend to align along the longitudinal orientation of the patterned stripe [21].

For a IrMn- or PtMn-based SAF structure, with an increasing annealing temperature, the exchange bias field decreases $[22,23]$. It may be beneficial for the alignment of the pinned layer easy axis along 
the transverse direction of the patterned elements because the induced anisotropy field is generally smaller than $\mathrm{H}_{\mathrm{ex}}$. The deviation of the pinning direction depends also on the value of the field applied during the thermomagnetic treatment [18]. In order to further optimize this full bridge sensor, a proper annealing condition needs to be exploited.

\section{Conclusions}

We have successfully designed and fabricated a novel angular GMR sensor in a full Wheatstone bridge by depositing one GMR film stack and utilizing simple patterned processes and a concise post-annealing procedure based on a special layout. The angular GMR sensor is of good linear performance and achieves a sensitivity of $0.112 \mathrm{mV} / \mathrm{V} /$ Oe at the annealing temperature of $260{ }^{\circ} \mathrm{C}$ in the magnetic field range from -50 Oe to +50 Oe. This work provides a design and method for GMR sensor manufacturing that is easy for implementation and suitable for mass production. The optimization of several aspects and more quantitative analyses are still needed in future work on this sensor.

Author Contributions: W.Z. and Q.L. supervised this work. S.Y. and Z.C. designed and performed the experiments. Z.Z. helped in the annealing step. S.Y. and Z.G. performed the measurements. Q.L. and S.Y. analyzed the data. Z.G. and A.C. contributed figure representations of this work. Y.Q. took part in the discussion of the work. S.Y. wrote the manuscript.

Funding: This work was financially supported by National Natural Science Foundation of China (No. 61627813), the International Collaboration Project B16001 and the VR innovation platform from Qingdao Science and Technology Commission.

Acknowledgments: The authors also would like to thank Li Huang from Institute of Physics, Chinese Academy of Sciences for the help on measurement work, Qiang Gui from Institute of Semiconductors, Chinese Academy of Sciences for the assistance with device fabrications and the engineers in Singulus Technologies for the cooperation in film stack deposition.

Conflicts of Interest: The authors declare no conflict of interest.

\section{References}

1. Baibich, M.N.; Broto, J.M.; Fert, A.; Vandau, F.N.; Petroff, F.; Eitenne, P.; Creuzet, G.; Friederich, A.; Chazelas, J. Giant magnetoresistance of (001) Fe/(001) Cr magnetic super lattices. Phys. Rev. Lett. 1988, 61, 2472-2475. [CrossRef] [PubMed]

2. Binasch, G.; Grunberg, P.; Saurenbach, F.; Zinn, W. Enhanced magnetoresistance in layered magnetic structures with antiferromagnetic interlayer exchange. Phys. Rev. B 1989, 39, 4828-4830. [CrossRef]

3. Wang, Z.; Wang, X.; Li, M.; Gao, Y.; Hu, Z.; Nan, T.; Liang, X.; Chen, H.; Yang, J.; Cash, S.; et al. Highly sensitive flexible Magnetic sensor based on anisotropic magnetoresistance effect. Adv. Mater. 2016, 28, 9370-9377. [CrossRef] [PubMed]

4. Cubells-Beltran, M.D.; Reig, C.; Madrenas, J.; De Marcellis, A.; Santos, S.; Freitas, P.P. Integration of GMR sensors with different technologies. Sensors 2016, 16, 939. [CrossRef] [PubMed]

5. Chen, Y.T.; Kolhatkar, A.G.; Zenasni, O.; Xu, S.J.; Lee, T.R. Biosensing using magnetic particle detection techniques. Sensors 2017, 17, 2300. [CrossRef] [PubMed]

6. Weiss, R.; Mattheis, R.; Reiss, G. Advanced giant magnetoresistance technology for measurement applications. Meas. Sci. Technol. 2013, 24,1-18. [CrossRef]

7. Lee, J.R.; Haddon, D.J.; Gupta, N.; Price, J.V.; Credo, G.M.; Diep, V.K.; Kim, K.; Hall, D.A.; Baecher, E.C.; Petri, M.; et al. High resolution analysis of antibodies to post-translational modifications using peptide nanosensor microarrays. ACS Nano 2016, 10, 10652-10660. [CrossRef] [PubMed]

8. Krishna, V.D.; Wu, K.; Perez, A.M.; Wang, J.P. Giant Magnetoresistance-based Biosensor for Detection of Influenza a Virus. Front. Microbiol. 2016, 7, 400. [CrossRef] [PubMed]

9. Dieny, B.; Speriosu, V.S.; Parkin, S.S.P.; Gurney, B.A.; Wilhoit, D.R.; Mauri, D. Giant magnetoresistance in soft ferromagnetic multilayers. Phys. Rev. B 1991, 43, 1297-1300. [CrossRef]

10. Coelho, P.; Leitao, D.C.; Antunes, J.; Cardoso, S.; Freitas, P.P. Spin valve devices with synthetic ferrimagnet free layer displaying enhanced sensitivity for nanometric sensor. IEEE Trans. Magn. 2014, 50, 4401604. [CrossRef] 
11. Chan, P.H.; Li, X.; Pong, P.W.T. Spin valves with Conetic based synthetic ferrimagnet free layer. Vacuum 2017, 140, 111-115. [CrossRef]

12. Cubells-Beltran, M.D.; Reig, C.; Munoz, D.R.; Freitas, S.I.P.; Freitas, P.J.P. Full Wheatstone bridge spin-valve based sensors for IC currents monitoring. IEEE Sens. J. 2009, 9, 1756-1762. [CrossRef]

13. Freitas, P.P. Spintronic sensors. Proc. IEEE 2016, 104, 1894-1918. [CrossRef]

14. Reig, C.; Ramirez, D.; Silva, F.; Bernardo, J.; Freitas, P. Design, fabrication and analysis of a pin-valved based current sensor. Sens. Actuators A Phys. 2004, 115, 259-266. [CrossRef]

15. Ueberschar, O.; Almeida, M.J.; Matthes, P.; Muller, M.; Ecke, R.; Ruckriem, R.; Schuster, J.; Exner, H.; Schulz, S.E. Optimized monolithic 2-D spin-valve sensor for high-sensitivity compass applications. IEEE Trans. Magn. 2015, 51, 40002404. [CrossRef]

16. Berthold, I.; Muller, M.; Klotzer, S.; Ebert, R.; Thomas, S.; Matthes, P.; Albrecht, M.; Exner, H. Investigation of selective realignment of the preferred magnetic direction in spin valve layer stacks using laser radiation. Appl. Surf. Sci. 2014, 302, 159-162. [CrossRef]

17. Kim, K.Y.; Jang, S.H.; Shin, K.H.; Kim, H.J.; Kang, T. Interlayer coupling field in spin valves with $\mathrm{CoFe} / \mathrm{Ru} / \mathrm{CoFe} / \mathrm{FeMn}$ synthetic antiferromangets. J. Appl. Phys. 2001, 89, 7612-7616. [CrossRef]

18. Miheev, M.N.; Naumova, L.; Chernshova, T.; Proglyado, V.; Kamensky, I.; Ustinov, V. Spin-Flop in synthetic antiferromagnet and anhysteretic Magnetic Reversal in FeMn-Based Spin Valves. IEEE Trans. Magn. 2016, 52, 2301104.

19. Tong, H.C.; Qian, C.; Miloslavsky, L.; Funada, S.; Shi, X.; Liu, F.; Dey, S. The spin flop of synthetic antiferromagnetic films. J. Appl. Phys. 2000, 87, 5055-5057. [CrossRef]

20. Redon, O.; Albuquer, G.B.; Rodrigues, L.M.; Silva, F.I.; Freitas, P.P. Annealing effect on spin-valve sensor transfer curves. IEEE Trans. Magn. 1998, 34, 562-567. [CrossRef]

21. Qian, Z.; Bai, R.; Yang, C.; Li, Q.; Sun, Y.; Huo, D.; Li, L.; Zhan, H.; Li, Y.; Zhu, J. Effective anisotropy field in the free layer of patterned spin-valve resistor. J. Appl. Phys. 2011, 109, 103904. [CrossRef]

22. Anderson, G.W.; Huai, Y.; Pakala, M. Spin valve thermal stability: The effect of different antiferromagnets. J. Appl. Phys. 2000, 87, 5275-5278. [CrossRef]

23. Kim, M.J.; Kim, H.J.; Kim, K.Y.; Jang, S.H.; Kang, T. The annealing effect on GMR properties of PtMn-based spin valve. J. Magn. Magn. Mater. 2002, 239, 195-197. [CrossRef] 